\title{
(息)
}

Citation:

Yeo, RK and Gold, J and Marquardt, MJ (2015) Becoming "leaderful": Leading forward in turbulent times. Industrial and Commercial Training, 47 (6). 285 - 292. ISSN 0019-7858 DOI: https://doi.org/10.1108/ICT-04-2015-0032

Link to Leeds Beckett Repository record:

https://eprints.leedsbeckett.ac.uk/id/eprint/1552/

Document Version:

Article (Accepted Version)

The aim of the Leeds Beckett Repository is to provide open access to our research, as required by funder policies and permitted by publishers and copyright law.

The Leeds Beckett repository holds a wide range of publications, each of which has been checked for copyright and the relevant embargo period has been applied by the Research Services team.

We operate on a standard take-down policy. If you are the author or publisher of an output and you would like it removed from the repository, please contact us and we will investigate on a case-by-case basis.

Each thesis in the repository has been cleared where necessary by the author for third party copyright. If you would like a thesis to be removed from the repository or believe there is an issue with copyright, please contact us on openaccess@leedsbeckett.ac.uk and we will investigate on a case-by-case basis. 


\section{Becoming 'Leaderful': Leading Forward in Turbulent Times}

\section{Structured Abstract}

Purpose: The primary aim of the paper is to offer a practice-based understanding of leadership based on the concept of 'leaderful' practice. In supporting this concept, the paper describes the contexts that shape leadership capacity and introduces an integrative framework that further illustrates 'leaderful’ practice.

Methodology: The paper draws on prior research conducted by the authors in a variety of industries. Insights were gleaned from both theoretical perspectives and qualitative data drawn from a number of empirical studies.

Findings: In order to lead confidently in turbulent times, leaders need to first unlearn the conventional wisdom of leadership. Three contextual enablers contribute to 'leaderful' practice, namely problem, action, and experience. Becoming 'leaderful' is being mindful of how these three enablers could be harnessed and integrated to facilitate change in meaningful ways.

Practical Implications: In order to promote ‘leaderful’ practice, both reflective and conversational spaces are imperative. Such spaces help leaders to be mindful of their internal and external contexts, including a keen awareness of self and others in framing references of the past for the future. In doing so, leaders need to be 'present' to confront 'wicked' problems and take action through collective experience and intelligence.

Originality/Value: Understanding how leaders think, feel, and act in actual practice helps us understand the genuine characteristics of leadership. The paper introduces a framework of 'leaderful' practice with a focus on leading with confidence. It extends current understanding of leadership practice by viewing 'leaderful' practice from the perspective of problem, action, and experience. 
Keywords: Problem, action, experience, context, 'leaderful’ practice

Paper Type: Research paper

\section{Suggested Box-Out Quotes:}

“A productive way of developing leadership competencies is to create opportunities for leaders to engage in real problems and provide the contexts for them to 'pause and think' as well as 'test and experiment'.”

"In a world of turbulence, it is necessary for leaders to be mindful of being authentic in their thoughts, emotions, and actions.”

"Mindfulness is facilitated by a deeper understanding of the interplay of problems that confront us, actions we develop, and experiences we share.”

"Being 'leaderful' is focused on the work or activity of the group rather than the decision and action of a single leader.” 


\section{Becoming 'Leaderful': Leading Forward in Turbulent Times}

\section{The changing role of leadership}

The role of leadership is no longer a straightforward practice in current volatile times. As many organizations become increasingly networked and complex with cross-border involvements and activities, leaders will not only need to adopt a global mindset but also be highly adaptive to meet unconventional demands from internal and external stakeholders. The success of any organizational transformation effort is contingent upon leaders who are capable of working through ambiguity and equipped with the capacity to learn continuously to meet ongoing challenges. Leaders therefore need to redefine their priorities, shape their expectations, and seek new contributions according to dramatic shifts in contexts. As such, leaders need to change the way they view and engage in their work to enhance and sustain their competitive edge.

In this paper, we adopt a practice perspective of conceptualizing leadership for turbulent times. This perspective focuses on the daily activities of leaders and how they develop decision making and problem solving skills to handle unexpected circumstances arising from their ongoing practice. Understanding how leaders think, feel, and act in moments of mindful practice helps us understand the more genuine characteristics of leadership. This way, we can explore deeper insights into leadership practice by understanding how leaders organize their daily activities and how they design their actions to achieve desired outcomes. The purpose of this paper is threefold: to offer a practice-based understanding of leadership through 'leaderful' practice; to determine the contexts that shape leadership capacity; and to propose an integrative perspective of leadership in turbulent times. 
We live in a world with different facets of problems and challenges. These can either be developmental or destructive - that is, providing opportunities for leaders to learn and grow or stagnate and disconnect. Everyday problems, regardless of how they are perceived, can provide opportunities for learning and experimentation. Problem solving and learning occur simultaneously and spontaneously in constant changing contexts (Kolb, 1983). As such, a productive way of developing leadership competencies is to create opportunities for leaders to engage in real problems and provide the contexts for them to 'pause and think' as well as 'test and experiment'.

\section{Becoming 'leaderful' in practice}

In a world of turbulence, it is necessary for leaders to be mindful of being authentic in their thoughts, emotions, and actions. When organizational environments are in a constant flux, leaders could serve as stabilizing agents by being 'leaderful' in their practice (Raelin, 2011). As opposed to being objective and rational on technical issues, 'leaderfulness' is about the intuitive and interpretive aspects of relational activities (Carroll et al., 2008). Simply put, leadership is situated in its activity or practice rather than demonstrated through the heroism of individual leaders. In contrast to conventional perspectives, 'leaderful' practice is about the organizing of leadership work or activities rather than the vision or mission leaders offer to their organizations. In particular, being 'leaderful' is to be aware of how and why leaders think, feel, and act the way they do in day-to-day activities. In order to help organize and order disruptive patterns of work in turbulent environments, leaders need to 'pause and think' about what is going on around them.

Leaders also need to harness and mobilize the intellectual capacity of their employees to develop a culture of continuous learning and participation. Employees serve as collaborative and change agents that enhance the overall scope and depth of participation in 
organizational activities. Being 'leaderful' calls for a deeper sensitivity towards multiple levels of experience embedded in these agents' contributions and participation. As such, leaders need to learn to grasp and transform experiences from the individual, team, organizational, and network levels, particularly the interconnections between them (Raelin, 2010). In doing so, ‘leaderful’ actors embrace learning as an inescapable of organizational life by allowing collective wisdom to be generated from bottom up rather than top down. This requires that they provide a psychologically safe space for employees to engage in open and honest conversations allowing shared objectives to develop (Friedrich et al., 2009).

'Leaderful' actors also promote autonomy amongst their employees by not only recognizing individual contributions but also collaborative outcomes achieved through interdependent engagements (Knowles, 1980). Creating the context for autonomous learning and practice transitions employees from 'pause and think' to 'test and experiment' in more productive ways. Testing and experimentation allow taken-for-granted assumptions to be challenged and provide opportunities for employees to take responsibility for and reflect on their own actions. 'Leaderful' actors facilitate the reconstruction of shared activities to create mutual interests (Raelin, 2011). In short, being 'leaderful' is focused on the work or activity of the group rather than the decision and action of a single leader.

\section{Shaping leadership capacity}

\section{Leading through problems}

Everyday problems, no matter how disruptive, could serve as catalysts for action and learning. The more complex and urgent the problem, the better it is at bringing out the critical behaviors of leaders (Marquardt, 2011). 'Wicked' problems, commonly surfaceed in turbulent times, are critical sources of learning as these are complex problems with multiple layers of interconnected issues (Grint, 2005). Organizing the apparent chaos therefore 
becomes daunting for most leaders. Often, a solution springs up as a potential problem that creates greater disorder in the overall problem context. Understanding the-problem types and the difference between problems and dilemmas will help leaders know the underlying issues and how these might be reframed to generate more thoughtful conversations and actions. For instance, reframing through broad and specific questions could help with the diagnosis of the problem in a wider and more in-depth manner.

A diverse understanding of the problem and its immediate context is crucial for leaders and organizational members to interpret their roles, tasks, and tools in more varied ways. In doing so, they challenge underlying assumptions through feedback loops and reflection to bring about a new level of understanding of work, particularly the meanings of ongoing activities. The 'problem' context offers the opportunity and capacity for leaders to create conversational and action spaces to explore and understand complex organizational phenomena (Marquardt and Yeo, 2012). For instance, leaders engage in the subjective and objective evaluation of organizational elements and dynamics that both enable and constrain their boundaries of influence. They further examine their values and goals in relation to internal and external conditions affecting their identity and power to act (Revans, 1982). Leaders could potentially be asking: What should be happening? What is stopping it from happening? What can I do to remove the blockage?

Problem-driven leadership involves a series of distinct but interrelated actions involving information seeking, assumption or theory formulation, experimentation of assumptions, evaluation of experimentation, and review of the gap between expectations and results. With both espoused theory (concept) and theory-in-use (action) tested in actual problems, leaders begin to develop a sharper awareness of their roles and the situations they attempt to influence (Argyris and Schön, 1996). Engaging in problems helps leaders to reframe the context through questioning and this in turn triggers more productive
Commented [J G1]: Leaders can easily adopt a problem/solution mentality. But with complex problems, often what seems like a solution can created greater disorder in the overall problem context.

Commented [J G2]: Dilemmas are often confused as problem/solution when what is needed is $a$ an understanding of alternative possibilities which are both required for progress. 
conversations with their members in talking out (defining 'how') and talking through (discussing 'why') various underlying issues. Problem-driven conversations promote feedback loops and critical reflection that help both leaders and their members to delve deeper into the symptoms but at the same time maintain a broader perspective of the impact of these issues on the wider context of the organization (Rudolph et al., 2009).

Leading through action

The problem-oriented nature of learning gives rise to distinct actions that leaders should be mindful of. Action is a powerful response to complex circumstances as it captures both the cognitive and behavioral aspect of one's ability to lead and learn. Action is the behavior behind one's thought process enabling leaders to turn abstract conceptualization into concrete experimentation (Kolb, 1984). In doing so, the action of a leader has the potential to challenge routines, norms, and even common assumptions creating a new context for doing things differently (Brown et al., 1989). As an extension to problem solving, the action process could be exploratory or assertive in nature. Either way, a leader's action could enable or constrain the actions of others preventing or promoting collaborative inquiry and experimentation.

Leadership action is powerful as it operates at both the micro and macro levels of organizational effectiveness. At the micro level, a leader could empower others to take action by 'transferring' the influence of power to those he/she trusts. In doing so, the leader creates new meanings in action structures where the empowered action becomes a source of network to achieve wider objectives. At the macro level, a leader could enact coproduction of actions by drawing on the know-how of his/her members to collectively interpret and cultivate a sense of mindfulness for responding to complex organizational issues. A repertoire of subactions is involved in a single action-taking process. For instance, action taking is characterized by mentally organizing the action steps, physically preparing, executing, 
anticipating a response from the environment, and developing an awareness of the potential outcomes (Weick, 1995).

When leaders allow their members to coproduce actions collectively, they also open the space up for others to make sense of the tasks and environment together. In the process, they exchange worldviews about the way their organizational reality is presented and how they experience it. The dynamics further influence them to shape each other's frames of references for developing interconnected and shared actions. Understanding each other's worldviews helps leaders enhance their decision making ability at various levels through procedural, structural, systems, and strategic development. Ultimately, when leaders master their 'learned' action - that is action that takes into consideration the dynamics of the environment in which the action is situated - they will in turn influence their members' learning-to-learn attitude. Collectively, leaders will help them develop the courage to unleash more powerful individual actions that will have an impact on others (Yeo and Gold, 2011).

\section{Leading through experience}

Experience is a powerful context through which one engages in one’s sensory system premised on how one thinks, feels, and acts (Dewey, 1938). However, experience could be explicit or implicit and direct or direct depending on the way one interprets one’s knowing and becoming. 'Knowing' suggests an implicit understanding of the fundamental rules that govern a context of which experience is constituted. For instance, in their day-to-day interactions with others, some leaders operate on the assumption of taken-for-granted social dynamics and hence may be less sensitive to the needs of others. Such leaders' sense of knowing, when engaged in an activity with others, may prevent them from experiencing the more concrete aspects of the context. On the other hand, 'becoming' suggests a more overt expression and awareness of one's role and a more spontaneous understanding of how that role might be shaped by the dynamics of a particular context. Of becoming is simply allowing 
oneself to make sense of one's role in relation to one's external world. For instance, when leaders seek to be part of a team, they become more sensitive towards relational nuances including the language and behavior of others, and how external factors contribute to the dynamics they are in.

Experiences are also interrelated in many ways, often times complex and difficult to grasp. Leaders who draw on their concrete experience to help them organize meanings surrounding their practice tend to develop curiosity towards the scope and depth of lessons learned in particular experiences (Kolb, 1983). An important aspect of experience is the variety of actions that leaders take to help them make sense of their roles, their relationship with others, and their connection with the wider organization. In order to lead through turbulent times, leaders need to be mindful of the interplay of knowing and becoming (Clegg et al., 2005) weaving in and out of explicit and implicit experiences to develop situated practice. Situated practice is determined by making decisions and taking appropriate actions in the moment or action at the right time (Cook and Brown, 1999). In other words, leaders' mindfulness of the repertoire of their prior experiences leads to sensitivity towards the dynamics that unfold in ongoing experiences. In doing so, they engage in feedback, dialogue, and reflection that facilitate their sensemaking towards their role, identity, and potential influence. Leaders who are 'experiential' - that is a heightened awareness of the scope and depth of a context - are skilled at negotiating the tension between their inner self and outer world as well as their logic and symbolic representation in order to fully grasp and transform an experience (Fenwick, 2003).

\section{Leading in turbulent times}

Leaders of tomorrow must be 'present' today. They need to be adaptive by being aware of context, adopt a design approach to action, and harness both prior and lived 
experience in order to respond to complexity. We therefore propose a framework of 'leaderful practice' (see Figure 1) in response to turbulent times, characterized by the intersection of problem, action, and experience which serve as contextual enablers. We suggest that emerging leaders should see 'wicked' problems as opportunities for learning about complexity, develop actions that facilitate change in a particular system as well as grasp and transform experience in order to develop innovative processes in organizations. In what follows, we will illustrate how the integrative framework can be a starting point for developing 'leaderful' practice. We further illustrate each contextual enabler through direct quotes drawn from interview data drawn from a number of empirical studies we have conducted.

\section{[Insert Figure 1 about here]}

First, leading through problem requires that 'leaderful' actors adopt a learning attitude towards organizing and managing complexity in problem contexts. For instance, a group of managers we studied in an international consulting firm suggested that "not all problems are to be solved"; instead, problems are disguised as "teachers for delivering critical lessons". Learning those lessons, as found, would require 'leaderful' actors to "seize the moment” to recognize urgency and "get others to talk about them [lessons]”. This firm created ‘conversation labs’ for a network of people including consulting partners, senior and junior consultants, and their clients to participate in "talking out loud”. Some leaders, while recognizing the importance of deliverables to their clients, were more mindful than others to "navigate the mess" and "learn to make sense” about different facets of the same problem. A striking characteristic of these 'leaderful' actors was that they adopted a participatory approach to engaging in the problems through various people. They helped "give sense" when "others failed to make sense of the situation”. Through sensegiving, they encouraged others to discover "grey areas... intertwined by internal and external factors" that might or 
might not be controlled by the firm. By capitalizing on problems, these leaders allowed their members and clients to co-create solutions that could only be shaped through ongoing conversations.

Second, leading through action requires that 'leaderful' actors develop timely and mindful actions that facilitate change. Not all people have a deeper awareness of the criticality of their actions and those of others, ignoring the possibility that action could also refer to cognitive activities like analytical thinking or idea generation. In a

Telecommunications company we studied where groups of managers were involved in action design to improve service delivery and customer satisfaction, we discovered that they engaged in visual interactions of their "ideas for action” via different teams involving engineers as well as marketing and customer service personnel. The visual representations through diagrams and graphs helped create a "storyboard of actions” in one’s mind. This process communicated "structures and their internal workings" allowing them to "see the big and small pictures”. The managers then facilitated conversations a level up by connecting the 'structures' to systems within the company such as IT, culture, knowledge management, and so on. As people "on the ground" tried to see and connect to things at another level, they developed a clearer understanding of their roles and the actions they would contribute to the organizational system. According to a senior manager, “It’s all a learning process as no structure or system is perfect. We are 'work in progress'.” As realized, actions, no matter how small, can have an effect on the wider structure and system of actions. 'Leaderful' actors encourage their teams to be curious about their action taking.

Third, leading through experience is about leaders learning to grasp and transform experience in order to innovate. The challenge is to integrate individual experiences into a collective synergy of experiences to respond to ongoing change. Grasping an experience is recognizing the "stories of others" and "connecting them into an organizational story", 
according to a manager of a pharmaceutical company we studied. The pharmaceutical industry is competitive across the globe and this company sought to harness the individual experiences of their employees who have a role in connecting to people outside the company such as healthcare, governmental, and trade institutions. The intent was to "learn from individual best practices” to help "increase work efficiency... and serve our customers and meet regulatory requirements better.” The lead pharmaceutical management team was interested in transforming the diverse individual experiences into different domains of best practices that could be institutionalized. Their starting point was to learn from one another and provide the space for cross-functional interactions where "the left hand should know what the right hand is doing....as both are parts of the same body.” Building a network of relationships between disparate teams proved to be daunting but these managers capitalized on company events such as product launches and corporate communication sessions to "bring everyone back to the table" as various activities were created for teams to "talk to one another”. Being 'leaderful' for the company was driving change through individual and collective experiences.

Finally, the integration of leading through problem, action, and experience is represented by "X”, as illustrated in Figure 1. 'Leaderful' practice in " $\mathrm{X}$ ” can be described as the mindfulness of self and others in the context of change. Such mindfulness, as argued, is facilitated by a deeper understanding of the interplay of problems that confront us, actions we develop, and experiences we share, as encapsulated in Table I. In turn, mastery of the interplay is necessary for us to lead confidently through turbulent times.

[Insert Table I about here]

\section{Leading forward}

This paper argues that in order to lead in turbulent times, leaders need to be adaptive by recognizing that expert or technical knowledge is no longer the driving force towards 
leading through complexity. Instead, leaders need to unlearn the conventional wisdom of leadership by shifting the locus of power to that of practice. By practice, we suggest that leadership is situated in the very activity of organizing and defining work. In doing so, leaders become more mindful of their roles in relation to the internal and external environments, motivating them to act more 'leaderfully'. Learning is a critical process of 'leaderful' practice. Not only are leaders more aware of emerging problems, the power of their actions, and the experiences they could grasp and transform but they are also mindful of the collective intelligence of others (Yeo and Gold, 2011). The paper demonstrates through an integrative framework supported by actual examples, suggesting that unless leaders engage in mindful conversations, reflections, and feedback with others, they will continue to lead with self-interest. The paper emphasizes 'leaderful’ practice as a critical pathway leading forward by creating the context for others to shape common objectives through collective practice. The more leaders unleash the power of who they are in relation to how they act, the more courageous they become in leading through turbulent times!

\section{References}

Argyris, C. and Schön. D. (1996), Organizational Learning II: Theory, Method, and Practice, Addison-Wesley, Reading, MA.

Brown, J. S., Collins, A. and Duguid, P. (1989), “Situated cognition and the culture of learning”, Educational Researcher, Vol. 18, No. 1, pp. 32-42.

Carroll, B., Levy, L. and Richmond, D. (2008), “Leadership as practice: Challenging the competency paradigm”, Leadership, Vol. 4, No. 4, pp. 363-379.

Clegg, S. R., Kornberger, M. and Rhodes, C. (2005), “Learning/becoming/organizing”, Organization, Vol. 12, No. 2, pp. 147-167. 
Cook, S. and Brown, J. S. (1999), "Bridging epistemologies: The generative dance between organizational knowledge and organizational knowing”, Organization Science, Vol. 30, No. 4, pp. 381-400.

Dewey, J. (1938), Experience and Education, Macmillan, New York, NY.

Fenwick, T. (2003), Learning through experience: Through orthodoxies and intersecting questions, Krieger Publishing, Malabar, FL.

Friedrich, T. L., Vessey, W. B., Schuelke, M. J., Ruark, G. A. and Mumford, M. D. (2009), "A framework for understanding collective leadership: The selective utilization of leader and team expertise within networks”, The Leadership Quarterly, Vol. 20, pp. 933-958.

Grint, K. (2005), “Problems, problems, problems: The social construction of 'leadership’”, Human Relations, Vol. 58, No. 11, pp. 1467-1494.

Knowles, M. (1980), The Modern Practice of Adult Education. From Pedagogy to Andragogy (2 ${ }^{\text {nd }}$ Ed.), Prentice Hall, Englewood Cliffs, NJ.

Kolb, D. A. (1983), “Problem management: Learning from experience”, in Srivastva, S. (Ed.) The Executive Mind, Jossey-Bass, San Francisco, CA, pp. 109-143.

Kolb, D. A. (1984). Experiential learning: Experience as the source of learning and development. New Jersey, NJ: Prentice-Hall.

Marquardt, M. J. (2011). Optimizing the Power of Action Learning ( $2^{\text {nd }}$ Ed.), Nicholas Brealey Publishing, Boston, MA.

Marquardt, M. J. and Yeo, R. K. (2012), Breakthrough Problem Solving with Action Learning: Concepts and Cases, Stanford University Press, Palo Alto, CA.

Raelin, J. A. (2010), The Leaderful Fieldbook: Strategies and Activities for Developing Leadership in Everyone, Nicholas Brealey, Boston, MA.

Raelin, J. A. (2011), “From leadership-as-practice to leaderful practice”, Leadership, Vol. 7, No. 2, pp. 195-211. 
Revans, R. (1982), The Origins and Growth of Action Learning, Chartwell-Bratt, Lund.

Rudolph, J. W., Morrison, J. B. and Carroll, J. S. (2009), “The dynamics of action-oriented problem solving: Linking interpretation and choice”, Academy of Management Review, Vol. 34, No. 4, pp. 733-756.

Weick, K. (1995), Sensemaking in organizations, Sage, Thousand Oaks, CA.

Yeo, R. K. and Gold, J. (2011), “The inseparability of action and learning: Unraveling Revans' action learning theory for HRD”, Human Resource Development International, Vol. 14, No. 5, pp. 511-526.

\section{Further reading:}

O’Connell, P. K. (2014), “A simplified framework for $21^{\text {st }}$ century leader development”, The Leadership Quarterly, Vol. 25, No. 2, pp. 183-203.

Yeo, R. K. and Marquardt, M. J. (2015), “(Re)Interpreting action, learning, and experience: Integrating action learning and experiential learning for HRD”, Human Resource Development Quarterly, Vol. 26, No. 1, pp. 81-107. 